\title{
Pulmonary tumour with high carcinoembryonic antigen titre caused by chronic propolis aspiration
}

\author{
W-C. Lin*, Y-T. Tseng ${ }^{\#}$, Y-L. Chang ${ }^{\top}$ and Y-C. Lee ${ }^{*,+}$
}

ABSTRACT: Carcinoembryonic antigen (CEA) titre elevation is sometimes found in benign diseases, such as gastro-intestinal tract inflammatory disease and chronic obstructive pulmonary disease; however, very high CEA titre is rarely encountered in benign pulmonary disease.

A 36-yr-old female, who had suffered from body weight loss, was found to have high serum CEA titre $\left(60.8 \mathrm{ng} \cdot \mathrm{mL}^{-1}\right)$. Image studies revealed one pulmonary tumour at the left lower lobe, satellite nodules and mediastinal lymphadenopathy.

Left lower lobectomy and lymph node dissection were performed for suspicious pulmonary malignancy. The pathological examination revealed that the tumourous lesion was composed of small and fragmented foreign bodies, fibrinopurulent exudate and heavy eosinophils. The bronchial epithelium was characterised by goblet cell hyperplasia and CEA overexpression. The remaining lung parenchyma possessed similar foreign body reaction. The patient's medical history was reviewed and it was found that she had spread propolis topically on nasal mucosa as an adjuvant therapy to asthma for 6 months prior to this medical event. The CEA titre decreased after the operation to 14.2 and $7.88 \mathrm{ng} \cdot \mathrm{mL}^{-1}$ after 2 weeks and 6 months, respectively.

Propolis is used widely in folk medicine but it also has strong sensitising potential. One rare case of propolis aspiration is reported with presentation mimicking lung cancer.

\section{KEYWORDS: Aspiration, carcinoembryonic antigen, propolis}

arcinoembryonic antigen (CEA) was first discovered by Gold and Freedman in the 1960s and it led to the use of this tumour marker to monitor efficacy of treatment for malignancy and to detect recurrence. CEA titre is elevated in several benign pulmonary diseases, but it is rarely very high. WEBER and KeRTTULA [1] demonstrated transient, but significant, serum CEA titre elevation in pneumonias of different aetiologies, but they did not consider CEA as a common acute phase reactant. On reviewing the literature, the current authors found no report about pulmonary foreign body reaction associated with high serum CEA titre.

\section{CASE REPORT}

A 36-yr-old female, a nonsmoker with asthma, complained of a 6-kg body weight loss in 6 months in the regular obstructive pulmonary disease follow-up for asthma. The findings on physical examination were unremarkable except for thyroid goitre. Thyroid hormones were all within normal limits. Laboratory study results were normal except for blood eosinophilia $\left(2.34 \times 10^{9}\right.$ units $\left.\cdot \mathrm{L}^{-1}\right)$ and high serum CEA titre $\left(60.8 \mathrm{ng} \cdot \mathrm{mL}^{-1}\right)$. Serial studies for occult malignancy were performed and revealed only one possible focus: a tumourous lesion at the left lower lobe of the lung, which was revealed after chest radiography and computerised tomography (CT). Mediastinal lymph node enlargement was also noted (fig. 1). Sputum culture did not yield any pathogen. Fibreoptic bronchoscopy was suggested but it was refused by the patient. Although CT-guided fine needle aspiration cytology examination of the tumourous lesion did not find any malignant cells, malignancy could not be completely excluded. After discussion with the patient, direct surgical resection was preferred. Standard left lower lobectomy and lymph node dissection were performed smoothly.

Within the resected pulmonary lobe, there was one brown, jelly-like mass, measuring $4.5 \times 2.2 \times 2.0 \mathrm{~cm}$
AFFILIATIONS

*Division of Thoracic Surgery, Dept of Surgery, and Depts of "Pathology, and +Traumatology, National Taiwan University Hospital and National Taiwan University College of Medicine, Taipei, and \#Dept of Surgery, National Taiwan University Hospital Yun-Lin Branch, Yun-Lin, Taiwan.

CORRESPONDENCE

Y-C. Lee

Division of Thoracic Surgery Depts of Surgery and Traumatology National Taiwan University Hospital 7 Chung San South Road 10002

Taipei

Taiwan

Fax: 886233933989

E-mail:wuj@ha.mc.ntu.edu.tw

Received:

October 312006

Accepted after revision:

March 212007

STATEMENT OF INTEREST

None declared. 

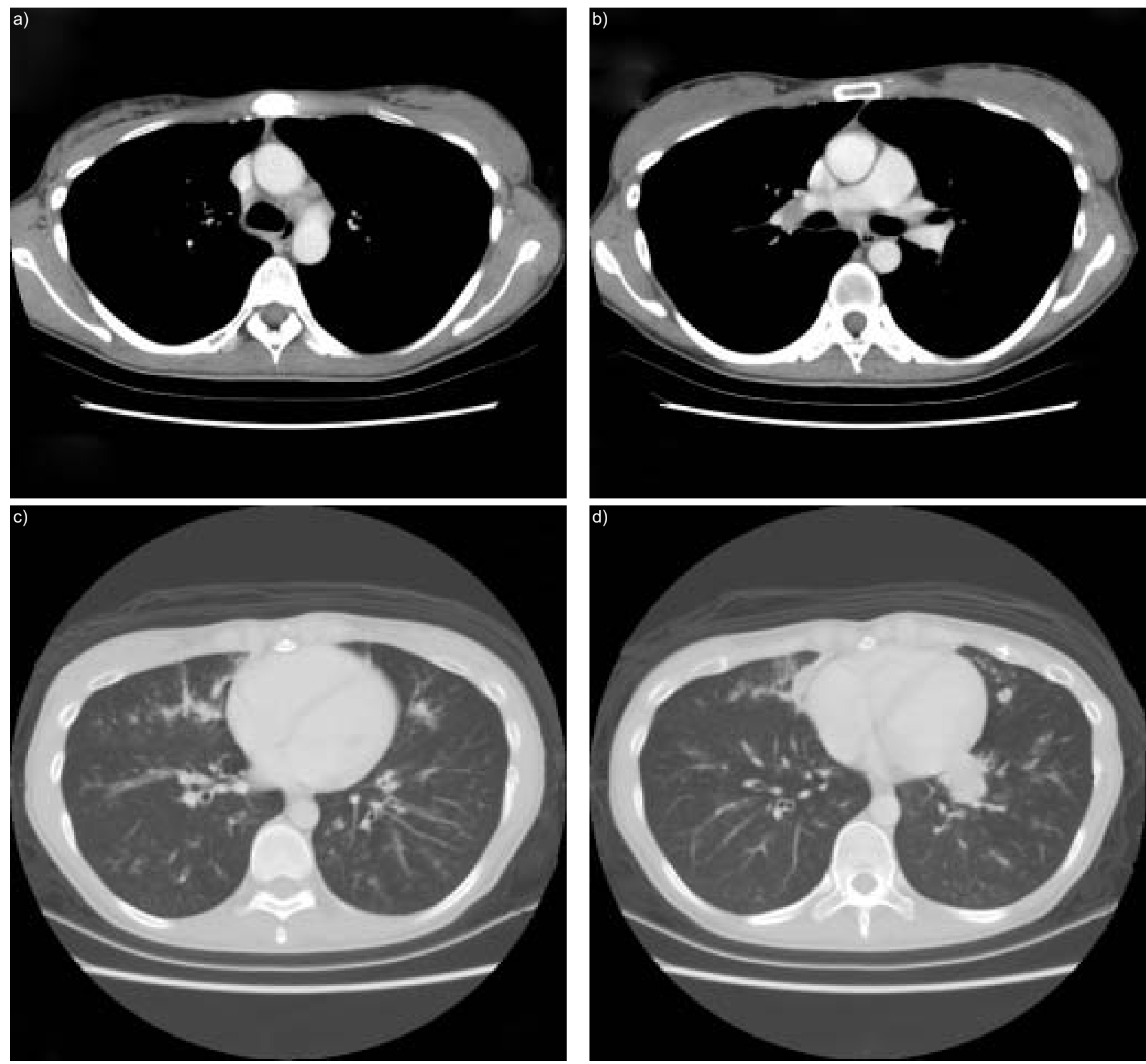

FIGURE 1. Computerised tomography of the chest showed a) lymphadenopathy at subaortic and b) subcarinal space, c) multiple small nodules at bilateral lungs, and d) one tumour with irregular margin at the left lower lobe.

in size, distal to the left lower lobe bronchus. Microscopically, the mass was composed of small and fragmented foreign bodies, fibrinopurulent exudates and heavy eosinophils. The bronchial epithelium showed prominent goblet cell hyperplasia accompanied by eosinophil infiltration (fig. 2a). The hyperplastic goblet cells were immunoreactive to CEA at the apical and basolateral regions. The CEA expression was also noted in the secretion within the bronchial lumen (fig. 2b). In the remaining lung parenchyma, there were scattered foci of foreign body aspiration (fig. 2c). No specific microorganism could be found by the periodic acid-Schiff and Gomori methenamine silver stains. All the mediastinal and lobar lymph nodes revealed reactive lymphoid hyperplasia with eosinophil infiltration.
Since the pathological report showed foreign body aspiration, the patient's medical history was reviewed. She had suffered from asthma and allergic rhinitis since childhood. In addition to modern medical treatment, she also sought Chinese folk medicine as an adjuvant therapy: she had smelled and inhaled liquid bee glue via the nasal mucosa for a 6-month period prior to the present event. She had also received inhalant allergenspecific immunoglobulin (Ig)E assays (Pharmacia CAP system) for common allergens in Taiwan, but no specific allergen was ever discovered.

After the operation, the CEA titre dropped dramatically to 14.2 and $7.88 \mathrm{ng} \cdot \mathrm{mL}^{-1}$ after 2 weeks and 6 months, respectively. 

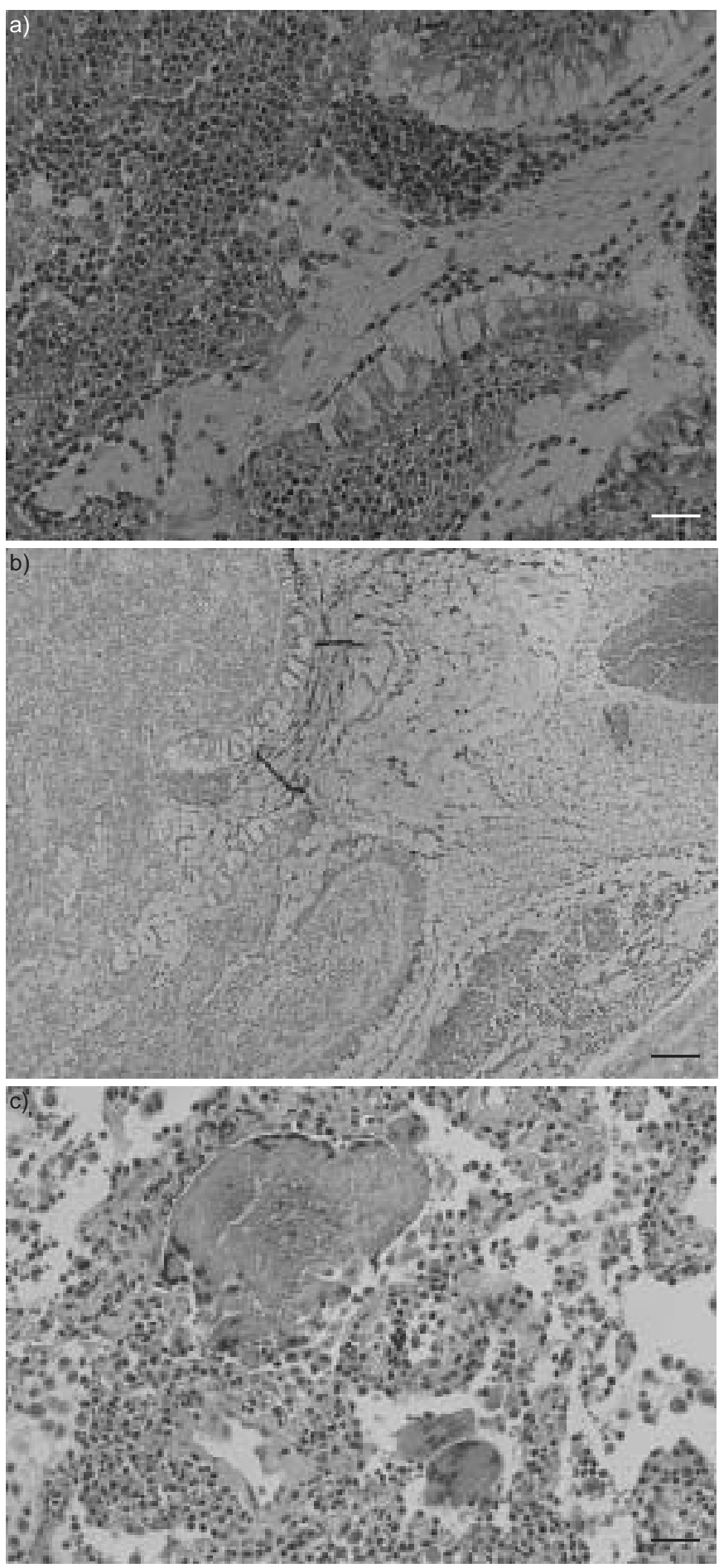

FIGURE 2. a) The bronchial epithelium showed prominent goblet cell hyperplasia accompanying with heavy eosinophil infiltration (haematoxylin-eosin $(\mathrm{HE}))$. b) Carcinoembryonic antigen immune reactivity was expressed at the basolateral and apical regions of goblet cells and secreted into bronchial lumen (avidin-biotin peroxidase complex method). c) The lung parenchyma revealed scattered foci of foreign body aspiration (HE). Scale bar $=1 \mathrm{~mm}$.

Eosinophil count also dropped to $0.46 \times 10^{9}$ units $\cdot \mathrm{L}^{-1}$. No elevation of either of these levels was found during the $2-\mathrm{yr}$ follow-up.

\section{DISCUSSION}

In the patient, appearance of pulmonary tumour and detection of high CEA titre occurred 6 months after commencing the administration of bee glue as adjuvant therapy for asthma. Besides, the aspirated foreign bodies were yellow to brown in colour, jelly-like, not soluble in water and diffusely distributed in the whole resected lobe of lung. These characteristics of the foreign bodies resembled those of the bee glue. The sequence of medical events also supports the suspicion that the pulmonary tumour was mainly composed of aspirated bee glue.

Bee glue, also known as propolis, is a resinous natural product elaborated by honey bees. It normally consists of 30\% wax, $55 \%$ resins and balsams, $10 \%$ essential oils and $5 \%$ pollen in addition to biologically active constituents. Propolis has been widely used in folk medicine and biopharmacological products for its antiviral, antimicrobial and anti-inflammatory activities. These activities are partially associated with inhibition of prostaglandins and leukotrienes [2, 3].

KHAYAAL et al. [2] demonstrated that propolis is an effective adjuvant to therapy in asthmatic patients. A marked reduction in the incidence and severity of nocturnal attacks and an improvement of ventilatory functions were found in mild-tomoderate asthmatic patients who use propolis as an adjuvant to oral theophylline therapy.

However, propolis also has strong sensitising properties and is well documented as an occupational contact allergen. Due to the increased use of propolis in folk medicine and the biocosmetic industry, nonoccupational contact allergy to propolis has risen recently [3]. Sometimes the pollen within the propolis products also plays a role in sensitisation. The case-report patient had negative allergen-specific IgE assay (Pharmacia CAP system) results for all common inhalant allergens in Taiwan, including pollen, before and after this medical event. Indeed, pollen is a less common and less significant allergen in Taiwan.

The patient had high serum CEA titre but the titre levels dropped after resection of the main lesion. Does airway allergic reaction to foreign bodies lead to high serum CEA titre? In adults, CEA modulates intercellular adhesion and functions as a promoter of cellular aggregation. It is usually overexpressed on the cell surface of malignant epithelial-type tumours and it may offer a survival advantage by allowing adhesion into other cells and further metastasis.

CEA titre elevation was also noted in benign inflammatory conditions of the respiratory or gastro-intestinal tract, but in these conditions the titre is usually $<10 \mathrm{ng} \cdot \mathrm{mL}^{-1}$. The literature was reviewed for benign conditions associated with a high CEA titre. ASHITANI et al. [5] reported a case of eosinophilic pneumonia associated with $17 \%$ eosinophilia in blood and a high serum CEA titre $\left(17.1 \mathrm{ng} \cdot \mathrm{mL}^{-1}\right)$. SCHOTTKE and PADBERG [6] also reported a female that presented extensive polypoid eosinophilic sinusitis with skull bony destruction and had remarkably high serum CEA titre levels $\left(85 \mathrm{ng} \cdot \mathrm{mL}^{-1}\right)$. Both cases had proliferation of goblet cells on the sinus or bronchial mucosa. Immunohistochemical stains revealed CEA overexpression on the surface of goblet cells and in the secretion 
within the lumen. This is the same phenomenon that was observed in the case-report patient.

CEA is one of the members of the Ig superfamily, which includes intercellular adhesion molecule (ICAM)-1, ICAM-2 and vascular cell adhesion molecule-1 [7]. These adhesion molecules play an important role in asthma and allergic reactions. Severe allergic reaction provokes increased adhesion molecules expression, which may include CEA.

The differences in CEA expression between normal and cancer cells include the pattern of glycosylation and localisation [8]. In normal or well-differentiated carcinoma cells, CEA distributes mainly on the luminal epithelium. In contrast, poorly differentiated carcinoma cells contain abundant CEA within the cytoplasm [9]. The tissue CEA staining patterns had been well studied and reported to be a prognostic indicator for colorectal and pulmonary adenocarcinoma [10,11]. NG et al. [10] classified the staining patterns into four types and demonstrated that the basolateral localisation of CEA was associated with more frequent vascular invasion and, therefore, high serum CEA titre.

Due to chronic asthma, the airway in the patient was characterised by epithelial damage, eosinophilia and goblet cell hyperplasia. Propolis aspiration induced acute exacerbation of these histological changes. Although this was a benign process, the bronchial epithelium showed excessive CEA expression not only on the apical but also the basolateral region of goblet cells. This staining pattern may cause CEA secretion into both bronchial lumen and vessels. Approximately 2 weeks after resection of the main lesion (equivalent to the half-life of CEA), the patient's serum CEA titre was re-checked and it had dropped dramatically. The reasons for the rapid decrease may be the removal of large amounts of allergens and the use of antihistamine agent and prednisolone. However, the remaining pulmonary lobes could also contain propolis according to pre-operative image findings. Due to potential minor foreign body reactions, the patient's serum CEA titre fluctuated $\left(7.88-16.2 \mathrm{ng} \cdot \mathrm{mL}^{-1}\right)$ during the first year of follow-up. The residual small amount of propolis was expectorated gradually with cease of foreign body reaction and the CEA titre returned to the normal range within $1 \mathrm{yr}$ after the operation.

For a speculative lung nodule with high serum carcinoembryonic antigen titre, there are several aetiologies besides malignancy. It should be kept in mind that strong airway foreign body reaction may manifest itself in a similar manner to lung cancer. In addition, since propolis has high sensitising potential, propolis products should be used more carefully.

\section{REFERENCES}

1 Weber TH, Kerttula Y. Carcinoembryonic antigen (CEA) in blood in cases of pneumonia. Scand J Infect Dis 1986; 18: 547-550.

2 Khayyal MT, el-Ghazaly MA, el-Khatib AS, et al. A clinical pharmacological study of the potential beneficial effects of a propolis food product as an adjuvant in asthmatic patients. Fundam Clin Pharmacol 2003; 17: 93-102.

3 Giusti F, Miglietta R, Pepe P, Seidenari S. Sensitization to propolis in 1255 children undergoing patch testing. Contact Dermatitis 2004; 51: 255-258.

4 Hsu CY, Chiang WC, Weng TI, Chen WJ, Yuan A. Laryngeal edema and anaphalactic shock after topical propolis use for acute pharyngitis. Am J Emerg Med 2004; 22: 432-433.

5 Ashitani J, Sakamoto A, Maki H, et al. [A case of eosinophilic pneumonia with elevated levels of carcinoembryonic antigen (CEA).] Nihon Kyobu Shikkan Gakkai Zasshi 1994; 32: 1194-1198.

6 Schottke H, Padberg BC. [Differential diagnosis of destructive processes of the skull base. Aggressive polypoid eosinophilic pansinusitis with expression of carcinoembryonic antigen (CEA).] HNO 1990; 38: 170-173.

7 Goldstein MJ, Mitchell EP. Carcinoembryonic antigen in the staging and follow-up of patients with colorectal cancer. Cancer Invest 2005; 23: 338-351.

8 Garcia M, Seigner C, Bastid C, Choux R, Payan MJ, Reggio $H$. Carcinoembryonic antigen has a different molecular weight in normal colon and in cancer cells due to N-glycosylation differences. Cancer Res 1991; 51: 5679-5686.

9 O'Brien MJ, Zamcheck N, Burke B, Kirkham SE, Saravis CA, Gottlieb LS. Immunocytochemical localization of carcinoembryonic antigen in benign and malignant colorectal tissues. Assessment of diagnostic value. Am J Clin Pathol 1981; 75: 283-290.

10 Ng IO, Ho J, Pritchett CJ, Chan EY, Ho FC. CEA tissue staining in colorectal cancer patients-correlation with plasma CEA, histology and staging. Pathology 1993; 25: 219-222.

11 Sawabata N, Hirano H, Inoue M, et al. Immunohistochemical analysis of resected clinical stage I pulmonary adenocarcinomas with high preoperative levels of serum carcinoembryonic antigen. Ann Thorac Surg 2003; 76: 203-207. 